\title{
SMUTS AND RUSTS OF NORTHERN UTAH AND SOUTHERN IDAHO
}

\author{
George L. ZundeL
}

The fungi listed in the following paper were secured from two principal sources. In the first place the author collected a number of smuts and rusts while on his vacation during August, I920. The next source of material was the herbarium of Dr. C. N. Jensen, formerly plant pathologist of the Utah Experiment Station at Logan, Utah. This material was secured from the biology department of the Brigham Young College at Logan, Utah. Besides the above two sources of material, miscellaneous collections that have been sent to the author have also been included. In some instances these miscellaneous collections are outside of Northern Utah or Southern Idaho. Where no credit is given it is the author's own collection.

The papers on the Smuts and Rusts of Utah by Prof. O. A. Garrett have been consulted in the preparation of this paper.

The author found that in Box Elder County, Utah, Ustilago bromivora was attacking Bromus tectorum in epiphytotic form. As a boy the author botanized in this section of the State without seeing this smut. In August, I920, however, he found hundreds of acres infected with this smut with an average infection of from 98 per cent to 99 per cent.

\section{SMUTS}

Tilletia asperifolia E1. \& Ev.; Jour. Myc. $3: 55$. i 887

On Sporobolus asperifola (Ness. \& Mey.) Thurb. At corner of $3 \mathrm{~d}$ North Street and 2d East Street, Logan, Cache County, Utah, August 3, I920 ( $9 \&$ per cent infection); a quarter mile north of Oregon Short Line Railroad depot, Logan, Cache County, Utah, August 4, I920 (98 per cent infection); West of Logan, Cache County, Utah, on Oregon Short Line Railroad, August 4, I920 ( 85 per cent infection); near Utah-Idaho Central Railroad, Five Points, Weber County, Utah, August I4, 1920 (98 per cent infection); one mile south of Brigham City, Box Elder County, Utah, August I6, I920 (75 per cent infection); Perry, Box Elder County, Utah, August 18, 1920 (infection only a trace). 
Tilletia foetans (B. \& C.) Trel. Par. Fungi, Wisc. $35 . \quad$ i 884

On Triticum sp. (cultivated wheat); Beaver, Beaver County, Utah, August 2I, 1918 (H. A. Christiánsen); Monticella, San Juan County, Utah, September 1918 (C. O. Stott); Kanab, Kane County, Utah, August 1918 (Hugh Hurst); Cedar City, Iron County, Utah, September I918 (Alma Esplin); Aberdeen, Bingham County, Idaho, September 8, I918.

Tilletia tritici (Bjerk.) Wint.; Rab, Kryp. Fl. i: i io. i 88 I

On Triticum sp. (cultivated wheat), Loa, Wayne County, Utah, September, I918 (A. E. Smith); North Logan, Cache County, Utah, August 5, 1920 ; American Falls, Power County, Idaho, September 9, 1918; Rockland, Power County, Idaho, September 9, 19г8; Winchester, Lewis County, Idaho, August 28 , 19 I 8 .

Ustilago bromivora (Tul.) Fisch. de Waldh. Bull. Soc. Nat. Mosc.

$$
40^{1}: 25^{2} \text {. I } 867
$$

On Bromus marginatus Nees., Mountain south of canyon road, Logan, Cache County, Utah, August io, igiz (C. N. Jensen, No. 238).

On Bromus tectorum L., Utah Agricultural College Campus, Logan, Cache County, Utah (85 per cent infection; Zundel \& Richards) August 3, 1920 ; Utah Agricultural College Campus, main entrance, Logan, Cache County, Utah, August 3, I920 (75 per cent infection); mouth of Logan Canyon, Cache County, Utah, August 4, 1920 (20 per cent infection); one mile up Logan Canyon, Cache County, Utah, August 4, 1920 (90 per cent infection); North Logan, Cache County, Utah, August 5, 1920 (4 per cent infection); Logan Canyon at Birch Glen, Cache County, Utah, August 7, 1920 (50 per cent infection); Logan Canyon at Rick's Spring, Cache County, Utah, August 7, 1920 ( 50 per cent infection); Ideal Beach on Bear Lake, Rich County, Utah, August 7 and 8, I920 (3 per cent infection); West of Newton, Cache County, Utah, August II, $1920^{\circ}$ (30 per cent infection); Clarkston, Cache County, Utah, August II, 1920 ( 15 per cent infection); Wandamere Park, Salt Lake City, Salt Lake County, Utah, August 13, 1920 (90 per cent infection); University of Utah Campus, Salt Lake City, Salt Lake County, Utah, August I3, 1920 (98 per cent infection); Lagoon Resort, Farmington, Davis County, Utah, August I4, I920 (8 per cent infection); Five Points, Weber County, Utah (less than I per cent infection); Reservoir Hill, Brigham City, Box Elder County, Utah, August I6, I920 (80 per cent to 99 per cent infection); South on Utah Idaho Central Railroad, Brigham City, Box Elder County, Utah, August 16, 1920 (85 per cent infection); Box Elder Creek, North of Brigham City, Box Elder County, Utah, August I7, I920 (95 per cent infection); Perry, Box Elder County, Utah, August I8, 1920 ( 8 per cent infection); Fish Haven, Bear Lake County, Idaho, August. 8, 1920 ( 2 per cent to 99 per cent infection); Tyhee, Bannock County, Idaho, August 19, I920 (trace of infection); ror4 W. Fremont Street, Pocatello, Bannock County, Idaho, August 20, 1920 (I5 per cent infection); Hills East of Pocatello, Bannock County, Idaho, August 20, 1920 (io per cent infection). 
Ustilago hordei (Pers.) Kel. \& Swing., Ann. Rep. Kans. Agr. Exp. Sta. 2: 268. 1890

On Hordeum sp. cult., Beaver, Beaver County, Utah (Christiansen). Ustilago LOREnTziana Thum. Flora 63: 30 . 1880

On Hordeum jubatum L. Logan, Cache County, Utah, August 5, I9I2 (Jensen No. 220); Beaver, Beaver County, Utah, August, I9I7 (Christiansen); Logan, Cache County, Utah, August 3, 1920 (90 per cent infection); Logan Canyon, Cache County, Utah, August 7, 1920 (5 per cent infection); Ideal Beach near South end of Bear Lake, Rich County, Utah, August 8, 1920 (30 per cent infection); Bloomington, Bear Lake County, Idaho, August 8, 1920 (8 per cent infection); Tyhee, Bannock County, Idaho, August i9, 1920 (trace of infection); East Halliday Street, Pocatello, Bannock County, Idaho, August 20, I920 (3 per cent infection); near subway, O. S. L. R. R., Pocatello, Bannock County, Idaho (25 per cent infection).

Ustilago longissima (Snow.) Tul. Ann. Sci. Nat. imi, 7: 76. 1847

On leaves of Glycera grandis Wats.-Banks of Logan River, West of Logan, Cache County, Utah, August 17, 1912 (Jensen No. 251).

Ustilago macruspora Desmaz. Pl. Crypt. II. i727. I850.

On leaves of Elymus canadensis L., Oregon Short Line Railroad West of Logan, Cache County, Utah, August 17, I9I2 (Jensen No. 250).

Ustilago tritici (Pers.) Rostr. Overs. K: Danske Vid. Selsk. Forh. I890: I5 Mch, I89o

On Triticum sp. (cultivated wheat) Greenville, Cache County, Utah, June 17, 1918; Beaver, Beaver County, Utah, August, I918 (Christiansen).

\section{RUSTS}

Aecidium abundans Pk. Bot. Gaz. $3: 34 . \quad$ i 878

On Symphoricarpus sp.-Mountains South of Canyon Road, Logan, Cache County, Utah, August ro, r9i2 (Jensen No. 237).

\section{Aecidium phalaris $\mathrm{Pk}$.}

On Phalaris leucophylla, Torr. Logan Canyon, Cache County, Utah, June 22, 1912 (Jensen No. 209).

Gymosporangium nelsoni Arth. Bull. Torr. Bot. Club 28: 665. igoi

I. On leaves of Amelanchier alnifolia Nutt. Dry Canyon, Cache County, Utah, August 2, igr2 (Jensen No. 277) ; Logan Canyon, Cache County, Utah. August 4, 1920.

Melamspora confluens (Pers.) Jack. Brook. Bot. Gard. Mem. I: 2 io. igi 8

II. On Salix sp.-Banks of Logan River, West of Logan, Cache County, Utah, August I5, I912 (Jensen No. 246); Logan Canyon, Cache County, Utah, August 7, 1920. 
Phragmidium affine Syd. Ann. Myc. 2: 29. 1904

I. On Potentilla gracilis Doug1. Logan Nursery, Logan, Cache County, Utah, June 24, 1912 (Col. Leo Merrill; Jensen No. 212).

Phragmidium imitans, Arth., N. A. Flora 7: i65. i9i2

III. On Rubus americana (Pers.) Wint.-Forks of Logan Canyon, Cache County, Utah, August i7, I9I2 (Coll. Zundel; Jensen No. 248).

II, III. On Rubus strigosus Michx. Forks Logan Canyon, Cache County, Utah, August I7, I9I2 (coll. Zundel; Jensen No. 249); Logan Canyon, Cache County, Utah, August 7, 1920.

Phragmidium nontivagum Arth. Torr. 9: i28. i 898

I, II. On Rosa sp. Dry Canyon, Cache County, Utah, August 2, I9I 2 (Jensen No. 224); Logan Canyon, Cache County, Utah, August 7, 1920.

Polythalis thalictri (Cler.) Arth. Résult. Sci. Cong. Bot. Vienne 341. igo6

On Thalictrum sp. Logan Canyon at Birch Glen, Cache County, Utah, August 7, 1920.

Puccinia arsinthil (Hedw. f.) DC. Fl. Fr. 6: 56. i8i5

II. On Artemesia bigelovia Gray, Providence Bench near Dry Canyon, Cache County, Utah, August 2, I9r2 (Jensen No. 225).

II, III. On Artemisia tridenta Nutt, Providence Bench, near planing mill, Cache County, Utah, August 2, 1912 (Jensen No. 226). Forks of Logan Canyon, Cache County, Utah, August 19, 1912, Tony Grove, Logan Canyon, Cache County, Utah, August 8, 1920.

Puccinia balsamiorrhizae Pk. Bull. Torr. Bot. Club ir: 49. is84

On Balsamorrhiza sagittata (Pursh.) Nutt, Logan Canyon, Cache County, Utah, August 4, 1920.

Puccinia comandrae Pk. Bull. Torr. Bot. Club il: 49. I884

III. On Commandra pallida DC. Logan Canyon, Cache County, Utah, June 22, 1912 (Jensen No. 21I).

Puccinia caricis (Schum.) Schröt. Krypt. Fl. Schles. 3: 327. i 889

II. On Carex aquatica Wahl. Fish Hatchery West of Logan, Cache County, Utah, August i3, I912 (Jensen No. 235).

Puccinia clematidis (DC.) Lagerh. Tromso Mus. Aarsh. i7: 47. i 895

I. On Clematis ligusticifolia Nutt, Logan Canyon, Cache County, Utah, August 7, 1920.

Puccinil intermixta Pk. Bot. Gaz. 4: 23i. 1879

On Iva axillaris Pursh. Perry, Box Elder County, Utah, August i8, 1920. (Det. H. S. Jackson.)

Puccinia Jonesii Pk. Bot. Gaz. 6: 226. i88i.

I. On Leptotaenia multifida Nutt. Logan Canyon, Cache County, Utah, May i6, igi2 (Jensen No. 207) and III. June 22, igi2 (Jensen No. 208). 


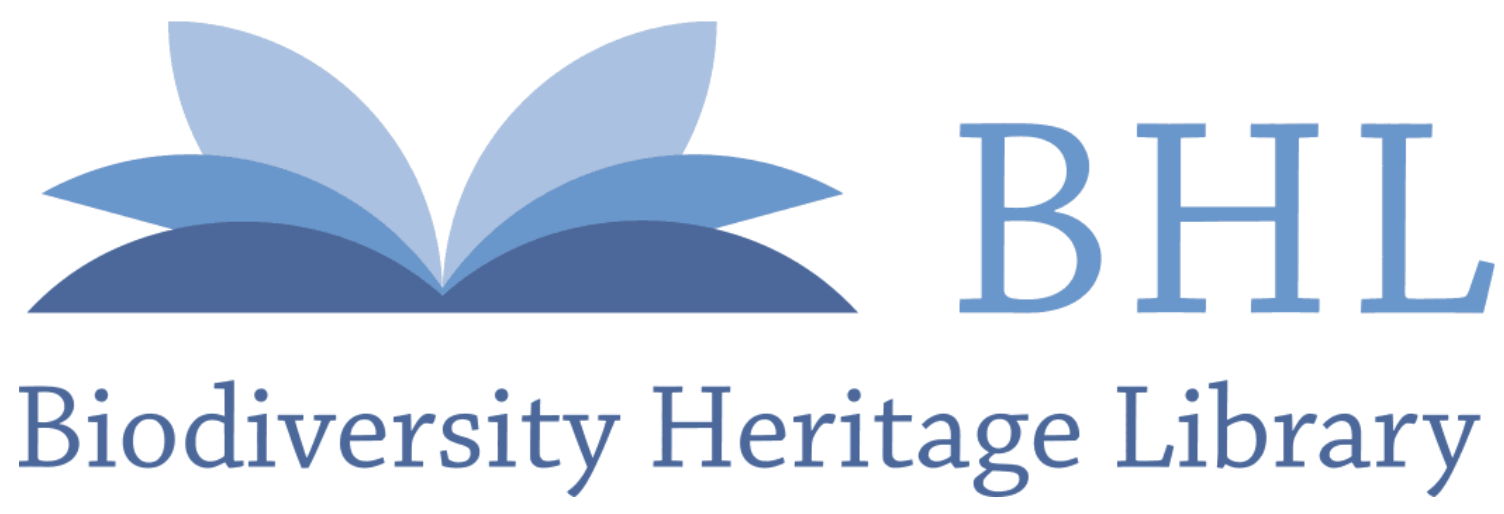

Zundel, George L. 1921. "Smuts and rusts of northern Utah and southern Idaho." Mycologia 13(3), 179-183.

View This Item Online: https://www.biodiversitylibrary.org/item/173510

Permalink: https://www.biodiversitylibrary.org/partpdf/246296

\section{Holding Institution}

Smithsonian Libraries

\section{Sponsored by}

Biodiversity Heritage Library

\section{Copyright \& Reuse}

Copyright Status: Not in copyright. The BHL knows of no copyright restrictions on this item.

This document was created from content at the Biodiversity Heritage Library, the world's largest open access digital library for biodiversity literature and archives. Visit BHL at https://www.biodiversitylibrary.org. 